\title{
Study of Congestion Control in Multi-Flow in MANET
}

\author{
Alok Kumar Dwivedi \\ Computer Science \& \\ Engineering \\ VNS Faculty of Engineering \\ Bhopal, India
}

\author{
Gaurishankar Prajapati \\ Headof the Department of Computer Science \& \\ Engineering \\ VNS Faculty of Engineering \\ Bhopal, India
}

\begin{abstract}
Mobile ad-hoc network is a next generation communication to provide cost free communication with independent decision making system, but now a day that is under research because number of pit fall in current scenario like route fluctuation, security and congestion, so all above problem solve with different technique base, here only resolve congestion problem with the explicit rate base congestion control method, in this paper provide all the exiting work done in the field of congestion control but here we proposed efficient and collaborative technique for that. In further we simulate our proposed work with NS-2 simulator and analyze the performance of the network.
\end{abstract}

\section{Keyword}

OLSR, CU, LQ, RBCC, XRCC, EXACT, TFRC.

\section{INTRODUCTION}

Nowadays mobile communication is used for various kinds of networked applications like service discovery [1], network discovery, automatic network reconfiguration etc. Many a time task processing is taken up by the agents that roam in the network and consequently execute the task it is responsible for. Thus nodes involved in task processing may not be needed to be connected throughout. This feature is a much desired one in resource constrained dynamic environments like Mobile Ad Hoc Network (MANET). Other benefits of using mobile agents include bandwidth conservation, reduced latency, load balancing etc.

The route of the mobile agent can be decided by its owner or it can decide its next hop destination on the fly. An agent may migrate in two ways, namely, weak migration and strong migration [2]. The platform is the environment of execution.

The platform makes it possible to create mobile agents; it offers the necessary elements required by them to perform their tasks such as execution, migration towards other platforms and so on. However, reliability and availability issues are needed to be addressed before mobile agent based (service discovery) protocols can be deployed for a broad range of commercial applications in MANET.

In mobile ad-hoc network communication case Congestion occurs when over a prolonged period of time more packets are generated than the network (as a whole or locally) can actually carry. Usually nodes have some buffer space available which can handle transient overloads. Any packet in excess of the available buffer space is dropped, wasting all the energy spent on this packet so far. Clearly. Tile larger this buffer space is the more overload can be carried and packet dropping occurs later. On the other hand longer queues impose longer end-to-end delays and the protocols need longer time to react on congestion states. Congestion control is very important in wireless sensor networks, because overloading a wireless network by too many transmissions can increase the collision probability. In this paper we explicit control the congestion with the help of rate estimation, queue limit and channel utilization threshold base mechanism, in the next section we provide related work in the field of congestion control and our proposed work to control new approach for congestion aware and control under MANET[3].

\section{RELATED WORK}

Makoto Ikeda et al [4] proposed "Congestion Control for Multi-flow Traffic in Wireless Mobile Ad-hoc Networks" in his work deploy the multi-flow scenario and apply OLSR with Link Quality (LQ) extension and fisheye-algorithm. The OLSR protocol is a pro-active routing protocol, which builds up a route for data transmission by maintaining a routing table inside every node of the network. The routing table is computed upon the knowledge of topology information, which is exchanged by means of Topology Control (TC) packets. The TC packets in turn are built after every node has filled its neighbors list. This list contains the identity of neighbor nodes. A node is considered a neighbor if and only if it can be reached via a bi-directional link. OLSR makes use of HELLO messages to find its one hop neighbors and its two hop neighbors through their responses. The sender can then select its Multi Point Relays (MPRs) based on the one hop node which offers the best routes to the two hop nodes. By this method, the amount of control traffic can be reduced. But that is implicit congestion control technique, in further extended that works with the help of channel utilization as well as queue length threshold base approach so explicitly congestion control.

Yong-Min Liu et al [5] proposed a DCCP (Datagram Congestion Control Protocol) they apply the four states four tcp congestion control: normal state, congestion state, failure state and error state with that technique they identify the congestion and resolve it. In this work researcher improve the throughput of the network.

Soundararajan et al [6] has on title "Multipath Load Balancing \& Rate Based Congestion Control for Mobile Ad Hoc Networks" in his approach contains an adaptive rate control based technique in which the destination node copies the estimated rate from the intermediate nodes and the feedback is forwarded to the sender through an acknowledgement packet. Since the sending rate is adjusted based on the estimated rate, this technique is better than the traditional congestion control technique. researcher apply the intermediate queue length threshold and channel utilization threshold base detection of congestion state and send feedback to the sender and congestion detected than sender set the new rate for data transmission.

S.Karunakaran et al [7] proposed a cluster based congestion control (CBCC) protocol that consists of scalable and 
distributed cluster-based mechanisms for supporting congestion control in ad hoc networks. The clusters autonomously and proactively monitor congestion within its localized scope. The present approach improves the responsiveness of the system when compared to end-to-end techniques. After estimating the traffic rate along a path, the sending rate of the source nodes is adjusted accordingly. Thus this protocol look forward the injection of dynamic flows in the network and proactively adjusts the rate while waiting for congestion feedback.

S.Venkatasubramanian et al [8] proposed quality of service architecture for Bandwidth Management and Rate Control in MANET. The proposed QoS architecture contains an adaptive bandwidth management technique which measures the available bandwidth at each node in real-time and it is then propagated on demand by the QoS routing protocol. The source nodes perform call admission control for different priority of flows based on the bandwidth information provided by the QoS routing. A rate control mechanism is used to regulate besteffort traffic, whenever network congestion is detected.

Kai Chen et al [9] proposed an explicit rate-based flow control scheme (called EXACT) for the MANET network. In EXACT, flow's allowed rate is explicitly conveyed from intermediate routers to the end-hosts in each data packet's special control header. As a result, EXACT reacts quickly and precisely to rerouting and bandwidth variation, which makes it especially suitable for a dynamic MANET network.

Kazi Chandrima Rahman et al [10] proposed explicit rate based congestion control (XRCC) for multimedia streaming over mobile ad hoc networks. XRCC addresses the problems that TCP faces when deployed over ad-hoc networks, and thus shows considerable performance improvement over TCP. Although XRCC minimizes packet drops caused by network congestion as compared to TCP congestion control mechanism, it still suffers from packet drops.

Hongqiang Zhai et al [11] proposed a novel rate based end-toend Congestion Control scheme (RBCC). Based on the novel use of channel busyness ratio, which is an accurate sign of the network utilization and congestion status, a new rate control scheme has been proposed to efficiently and reliably support the transport service in MANET. In RBCC, a sub layer consisting of a leaky bucket is added under TCP to control the sending rate based on the network layer feedback at the bottleneck node.

Emmanuel Lochin et al [12] proposed a complete reliable ratebased protocol based on TCP-Friendly Rate Control (TFRC) and selective acknowledgement (SACK) mechanisms.

This design also introduces a flow control variable, which regulates the sender to avoid packet loss at the receiver due to a slow receiver. In this mechanism, there is no packet loss due to flow control, at the receiver, and applies a smoothness criterion to demonstrate that the introduction of the flow control inside TFRC does not alter the smoothness property of this mechanism.

M Ali et. al. [13] in his proposed work "Congestion Adaptive Multipath Routing For Load Balancing In mobile Ad-hoc Networks "In this proposed work they present a congestion adaptive multipath routing protocol to increase the throughput and avoid congestion in MANET .when the average load of an existing link increases beyond a defined threshold and the available bandwidth and residual battery energy decreases below a defined threshold, traffic is distributed over fail-safe multiple routes to reduce the traffic load on a congestion.
Yuedong $\mathrm{Xu}$ et al [14] proposed a fully distributed congestion control algorithm to balance throughput and fairness for TCP flows in multi hop ad hoc networks. The interactions between the hidden nodes and network congestion are mainly focused. A distributed algorithm to improve the end-to-end throughput, and at the same time, provide per-flow fairness by exploiting cross-layer information is proposed. In the link layer, each node uses a proportional controller to determine the ECN marking probability for the purpose of notifying incipient congestion. Then the rate based TCP sender adjusts its sending rate according to the feedbacks from the link layer.

\section{OBJECTIVE}

Our aim is to design an efficient congestion control in multiflow traffic on the bases of intermediate node queue utilization and channel utilization technique with OLSR routing mechanism in mobile ad-hoc network, that explicit control the congestion from the network and minimize or avoid congestion with the help of feed backing message, after we decrease the routing overhead of the network and also increases packet delivery ratio of the network. Our protocol will ensure that there will be no dropping of packets in the network and hence ensure that there will be successful data transfer with lowest overhead required.

Importance of the research objective

Our research proposal is important for following purpose

Our objective to control the congestion for multi-flow traffic in dynamic environment.

Identify the congestion occurrence parameter like channel utilization, intermediate node load aware and queue utilization.

$>$ To provide the packet information such as data, routing or control packet and congestion dependent overhead are minimized.

$>$ Our objective to gives better throughput, packet delivery ratio with low overhead

$>$ It's provides reliable communication with efficient utilization of node capability.

Our proposed work also minimizes the end-to-end delay because explicit rate base congestion control dynamically changed the round trip time.

\section{MOTIVATION OF OUR WORK}

Mobile ad-hoc network is dynamic in nature with no centralize control because every node works on temporary bases and frequently changes their location, that is why we can't predicate that where the actual destination node is and what movement the nodes can take.

Some time it may happen that whole data or part of data is not reached at destination on time because of congestion or by other reasons in the network, since it is dropped in the network. When full data is collected on destination at their transport layer then some part of data get missed because it was dropped in network and at result end data is not fully assembled and finally it get corrupted. So we have a need of such a technology that can send the packet from explicit rate controlled manner by which if part of data is dropped at any hop, it will never cause loss at the end when it is assembled since it will reach from other hops in network and provide congestion free communication. 


\section{PROPOSED WORK}

Our proposed work to focus of controlling the congestion from the network with explicit rate measurement technique and minimize the congestion dependent wasteful packet flooding, for that purpose we divide our proposal into subpart and control the congestion as well as provide congestion free communication. while multiple source node after detecting path with the help of OLSR updated routing, they sends the data packet to the destination through the intermediate nodes but multiple source nodes share the common link that increase the congestion in between communication link. So here one extra header filed are added in the protocol architecture that gives the congestion information to the sender and explicit control the congestion, in before that we measure the congestion in each intermediate node on the bases of incoming and outgoing channel measurement and queue utilization of nodes, all node inbuilt the message of channel and queue utilization and forward to next hop that message decode by the receiver node and make decision for explicit control congestion information and send back acknowledgement to sender node, than sender explicit change data rate and provide congestion free communication.

The following step follows to congestion detection and control

\section{INCOMING AND OUTGOING RATE ESTIMATION}

The rate of the incoming and outgoing packet is estimated as follows.

The rate of the incoming packet $\left(\gamma_{i}\right)$ is estimated as the reciprocal of the arrival time interval of the incoming packet.

i.e. $\gamma \mathrm{i}=1 / \mathrm{Ti}$

$\mathrm{Ti}$ is defined as the time interval of two consecutive packets received at the node.

The rate of the outgoing packet $\left({ }^{\gamma} \mathrm{O}\right)$ is estimated as the reciprocal of the service time of the packet.

i.e. . ${ }^{\gamma} \mathrm{O}=1 /$ To

To is defined as the time interval between the time that a packet arrives at node and time that it is transmitted successfully. It is also defined as summation of the time for queue, collision, back off and transmission.

\section{(a) Estimation of Percentage Channel Utilization}

In the wireless mobile ad-hoc networks, when the transmission channel is being completely utilized, it is concluded that the network congestion has occurred. The channel utilization for time interval $t$ is estimated using channel busy-time $(\mathrm{Tc})$ metric on a percentage scale. The channel busy-time can be computed based on the category of control frame and the rate and data frame size.

The percentage channel utilization at time t, CU (t) [15] is given using $\mathrm{Eq}$ (3)

$$
\% C U=\left(\frac{T c(t)}{10^{6}}\right) * 100 .
$$

\section{(C) Estimation of intermediate Queue Length}

The queue length describes the total traffic load in a mobile node. In general, when excess traffic flows through the mobile node, then there will more number of packets in the interface queue. Thus average queue size (LQ) [16] is defined as the node's traffic in a long term. LQ is given using Eq (4)

$\mathrm{L}_{\mathrm{Q}}=\psi * \mathrm{~L}_{\mathrm{Qold}}+(1-\psi) * \mathrm{~L}_{\mathrm{Qc}}$

Where LQc $=$ current value of the queue length

$\psi=$ constant in the range $[0,1]$

\section{(d). Algorithm for Detecting Congestion}

1. The source sends the data packets to the destination through the intermediate nodes.

2. Let $\mathrm{L}_{\mathrm{Qth}}$ be the predefined threshold value of queue length. Let $\% \mathrm{CU}_{\text {th }}$ be the predefined threshold percentage channel utilization

Upon reception of the data packets, intermediate node verifies both the queue length and channel utilization

2.1 If $\mathrm{L}_{\mathrm{Q}}>\mathrm{L}_{\mathrm{Q} t h}$ and \%CU $>\% \mathrm{CU}_{\text {th }}$, Then

Set $C_{b}=1$

End if

2.2 If $\mathrm{L}_{\mathrm{Q}}<=\mathrm{L}_{\mathrm{Qth}}$ and $\% \mathrm{CU}<=\% \mathrm{CU}_{\text {th }}$, Then

Set $\mathrm{C}_{\mathrm{b}}=0$

End if

After the detection of congestion, the intermediate node estimates the new rate information. The source updates its packet sending rate with this estimated rate. This is done after getting acknowledgement packet from receiver as feedback regarding the nodes congestion status.

\section{(e) Working Architecture}

In this section describe the architecture of proposed technique where source node send the data packet to destination with the help of intermediate node, where each node contain the queue for incoming data storage that is meanly used where outgoing rate is less than the incoming data rate that is also control the congestion from the network, second property measure channel utilization between each node and set channel utilization threshold, both queue and channel utilization technique base we estimate the rate and send back the acknowledgment to send data in congestion controlled manner.

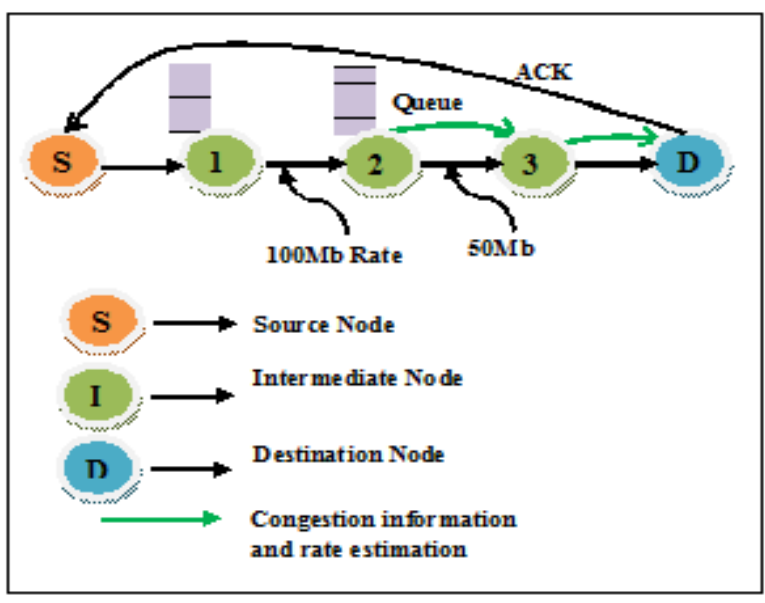

Figure 1: Working Architecture

\section{(f) Benefits of research work}

MANET has a dynamic network topology, and constraint 
resources, such as bandwidth, buffer space, battery and transmission power and so on. Distributing traffic fairly among the mobile hosts, based on measurement of path statistics, is beneficial in order to take full advantage of the limited resources and to use network resources better so that the congestion and end-to-end delay are minimized.

Purpose of explicit congestion control with rate estimation Schemes

The overall purpose of research is to:

1. Select non-congested paths or to minimize excessive load of a node to its neighbours.

2. Ensure efficiency and robustness.

3. Reduce end to end delay and number of packet lost by queue overflow.

4. Enhance the utilization of resources.

5. Improve the overall network performance and reduce collision by dynamic channel estimation base route selection.

\section{SIMULATION ENVIRONMENT}

Simulation will be done in Network Simulator- 2 (NS-2). The description about simulation environment is as follows:

Network simulator 2 (NS2) is the result of an on-going effort of research and development that is administrated by researchers at Berkeley [17]. It is a discrete event simulator targeted at networking research. It provides substantial support for simulation of TCP, routing, and multipath protocol.

The simulator is written in $\mathrm{C}++$ and a script language called OTcl2. Ns uses an Otcl interpreter towards the user. This means that the user writes an OTcl script that defines the network (number of nodes, links), the traffic in the network (sources, destinations, type of traffic) and which protocols it will use. This script is then used by ns during the simulations. The result of the simulations is an output trace file that can be used to do data processing (calculate delay, throughput etc) and to visualize the simulation with a program called Network Animator.

Network Animator (NAM)

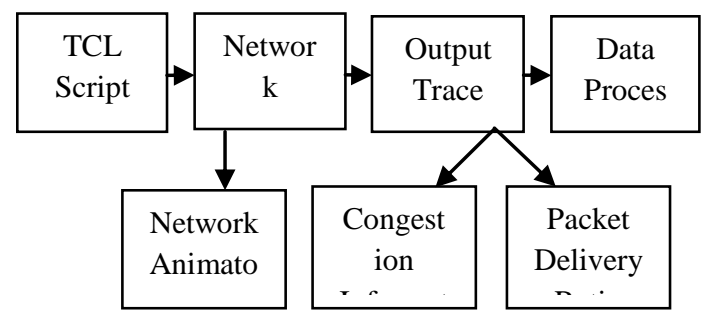

Figure 2: Network Animator

Script Awk

NAM is a very effective visualization tool that visualizes the packets as they propagate through the network. An overview of how a simulation is done in ns is shown in Figure 1.

\section{(a) Performance Measure}

Packet Delivery Ratio: The ratio between the number of packets originated by the application layer sources and the number of packets received by sink at the final destination.
Average End-to-end Delay: This includes all the possible delays caused by buffering during route discovery latency, queuing at the interface queue, retransmission delays at the $\mathrm{MAC}$, and propagation and transfer times.

Packet Dropped: The routers might fail to deliver or drop some packets or data if they arrive when their buffer are already full.

Routing Load: The total number of routing packets transmitted during the simulation.

\section{CONCLUSION}

In this paper investigate all the existing congestion control technique and identified their unique contribution in the field of congestion control after that we proposed newer explicit congestion control on the queue and channel utilization base sender rate estimation mechanism that objective to more efficient and robust method for overcome the congestion from the MANET network, in future we elaborate simulated experiment result and justified our proposed work so our contribution use full to daily life.

\section{REFERENCES}

[1] Roshni Neogy, Chandreyee Chowdhury, Sarmistha Neogy "A Reliable Service Discovery protocol using Mobile Agents in MANET" 978-1-4577-1851-9/12 IEEE

[2] N. Migas, W.J. Buchanan, K. McArtney, "Migration of mobile agents in ad-hoc, Wireless Networks", Proc.11th IEEE International Conference and Workshop on the Engineering of Computer-Based Systems, pp 530 - 535, 2004.

[3] Adel Gaafar A.Elrahim, Hussein A.Elsayed, Salwa El Ramly, Magdy M, Ibrahim "Improving TCP Congestion Control for Wireless Sensor Networks" 978-1-4577-09722/11 IEEE

[4] Makoto Ikeda, Elis Kulla, Masahiro Hiyama, Leonard Barolli, Rozeta Miho and Makoto Takizawa "Congestion Control for Mul i-flow Traffic in Wireless Mobile Ad-hoc Networks" 2012 Sixth International Conference on Complex, Intelligent, and Software Intensive Systems, 978-0-7695-4687-2/12 IEEE

[5] Yong-Min Liu, Xin-Hua Jiang, Xiao-Hong Nian, Wu-Yi $\mathrm{Lu}$ "Improved DCCP Congestion Control for Wireless Sensor Networks" 2009 Eigth IEEE/ACIS International Conference on Computer and Information Science, 978-07695-3641-5/09 IEEE

[6] Soundararajan et. al "Multipath Load Balancing \& Rate Based Congestion Control for Mobile Ad Hoc Networks (MANET)" 978-1-4673-0734-5/12 IEEE

[7] S. Karunakaran, P. Thangaraj, "A cluster based congestion control protocol for mobile ad hoc networks", International Journal of Information Technology and Knowledge Management, Volume 2, No 2, pp. 471-474, 2010

[8] S.Venkatasubramanian, N.P.Gopalan, "A quality of service architecture for resource provisioning and rate control in mobile ad hoc network", International Journal of Ad hoc, Sensor \& Ubiquitous Computing (IJASUC), Vol 1, No 3, September 2010

[9] K. Chen, K. Nahrstedt, N. Vaidya, "The Utility of Explicit Rate-Based Flow Control in Mobile Ad Hoc Networks", Proc. IEEE Wireless Communications and Networking Conference (WCNC 04), pp 1921-1926, 2004. 
[10] K. C. Rahman, S. F. Hasan, “ Explicit Rate-based Congestion Control for Multimedia Streaming over Mobile Ad hoc Networks", International Journal of Electrical \& Computer Sciences IJECS-IJENS, Vol 10, No 04,2010

[11] H. Zhai, X. Chen, Y. Fang, " Rate-Based Transport Control for Mobile Ad Hoc Networks", IEEE Wireless Communications and Networking Conference, pp 2264 2269, Vol 4, 2005

[12] E. Lochin, G. Jourjon, S. Ardon P. Senac, "Promoting the Use of Reliable Rate Based Transport Protocols: The Chameleon Protocol", International Journal of Internet Protocol Technology, Vol 5, Issue 4, pp 175-189, March 2010.

[13] M. Ali, B. G Stewart, A Shahrabi, A Vallavaraj “ Multipath Routing Backbones for Load Balancing in Mobile Ad Hoc Networks", 16th IEEE Mediterranean
Electro technical Conference on (MELECON), pp. 749 752,2012 .

[14] Y. Xu, Y. Wang, C.S. Lui, D-M. Chiu, "Balancing Throughput and Fairness for TCP Flows in Multi-hop AdHoc Networks", 5th International Symposium on Modeling and Optimization in Mobile Ad Hoc and Wireless Networks and Workshops (WiOpt), pp $1-10$, 2007

[15] P. Amit, N. Jardosh Krishna, C. Ramachandran Kevin, M. Almeroth Elizabeth, " Understanding Congestion in IEEE 802.11b Wireless Networks", In Proceedings of the 2005 Internet Measurement Conference, pp 158-169,2005.

[16] X. Gao, X. Zhang, D. Shi, F. Zou, W. Zhu, " Contention and Queue aware Routing Protocol for Mobile Ad hoc Networks", International Conference on Wireless Communications, Networking and Mobile Computing, (WiCom), pp 1628 - 1631, 2007. 